to multiple previous operations. ${ }^{1,2}$ In all these cases, and especially in case of PVE, the destruction of the architecture of the aortic root or the deficient aortic root tissues is a serious problem complicating aortic valve replacement, leading mortality rates as high as $20 \%$ to $30 \%{ }^{4,5}$

The eagle-shaped patch technique that we used in 2 cases of PVE, but that we believe could be considered even in the case of a small aortic root, is an easy and quick way to prevent the problems that may occur in the aortic-mitral areamalfunctioning of the mitral valve owing to overstretching of the anterior mitral leaflet and suture dehiscence owing to excessive tension on the reconstructed areas. Finally, with the "eagle" body, a complete reconstruction of the annulus and an easy and correct seating of the aortic prosthesis can be done.
Longer follow-up is obviously necessary to prove the effectiveness of our technique.

\section{References}

1. David TE, Kuo J, Armstrong S. Aortic and mitral valve replacement with reconstruction of the intervalvular fibrous body. J Thorac Cardiovasc Surg. 1997;114: 766-71; discussion 71-2.

2. David TE, Bos J, Christakis GT, Brofman PR, Wong D, Feindel CM. Heart valve operations in patients with active infective endocarditis. Ann Thorac Surg. 1990; 49:701-5

3. Mills SA. Surgical progress: surgical management of infective endocarditis. Ann Thorac Surg. 1982;195:367-83.

4. Vogt PR, von Segesser LK, Jenni R, Niederhauser U, Genoni M, Kunzli A, et al Emergency surgery for acute infective aortic valve endocarditis. Performance of cryopreserved homografts and mode of failure. Eur J Cardiothorac Surg. 1997; 11:53-61.

5. Edwards MB, Ratnatunga CP, Dore CJ, Taylor KM. Thirty-day mortality and longterm survival following surgery for prosthetic endocarditis: a study from the U.K. Heart Valve Registry. Eur J Cardiothorac Surg. 1998;14:156-64.

\title{
Totally thoracoscopic resection of a superior mediastinal tumor extending above the thoracic inlet
}

Toshiki Tanaka, MD, PhD, Kazuhiro Ueda, MD, PhD, Masataro Hayashi, MD, PhD, and Kimikazu Hamano, MD, PhD, Yamaguchi, Japan

The resection of superior mediastinal tumors extending above the thoracic inlet (extended superior mediastinal tumor) usually requires both transthoracic and transcervical approaches, either via thoracotomy in combination with a supraclavicular incision or via sternotomy, which imposes considerable stress on the patient. To reduce the excessive invasiveness, some surgeons have attempted to remove these tumors via video-assisted thoracic surgery, but this has always required simultaneous partial sternotomy or a supraclavicular incision. ${ }^{1,2}$ We report our initial experience of performing totally thoracoscopic resection of extended superior mediastinal tumors, without the need for transcervical access, in 2 patients. We believe that both technical and instrumental refinement contributed to the successful outcome of this challenging operation.

\footnotetext{
From the Department of Surgery and Clinical Science, Division of Chest Surgery, Yamaguchi University Graduate School of Medicine, Ube, Yamaguchi, Japan.

Disclosures: Authors have nothing to disclose with regard to commercial support.

Received for publication June 11, 2010; revisions received Aug 5, 2010; accepted for publication Aug 25, 2010; available ahead of print Nov 12, 2010.

Address for reprints: Toshiki Tanaka, MD, PhD, 1-1-1 Minami-kogushi, Ube, Yama-

guchi 755-8505, Japan (E-mail: toshik@yamaguchi-u.ac.jp).

J Thorac Cardiovasc Surg 2011;141:1323-5

$0022-5223 / \$ 36.00$

Copyright (c) 2011 by The American Association for Thoracic Surgery

doi:10.1016/j.jtcvs.2010.08.078
}

\section{CLINICAL SUMMARY}

Surgery is performed totally thoracoscopically via a 3-access port, eliminating the need for direct vision. The primary surgeon always stands to the right of the patient, and the assistant always stands to the left of the patient. The primary surgeon inserts the endoscopic instruments via the proximal 2 access ports on his/her side, and the assistant inserts the thoracoscope via the port on his/her side (Figure 1, $A$ ). The assistant simultaneously inserts the endoscopic dissector via the same port to create the best operating view.

\section{Patient 1}

A 67-year-old man was referred to Yamaguchi University hospital for surgical treatment of a superior mediastinal tumor. A chest computed tomography (CT) scan showed that the tumor comprised cystic and solid parts and was located in the right side of the superior mediastinum, extending to the cervical area just below the thyroid (Figure 2, A). Magnetic resonance imaging showed similar findings. These findings mimicked a mediastinal thyroid tumor. We attempted a total thoracoscopic resection of the tumor. Meticulous manipulation was necessary to dissect the feeding vessels from the inferior thyroidal artery and vein, and preserve the vagal nerve and recurrent nerve adjacent to the tumor in the thoracic inlet. The feeding vessels were divided, and the tumor was 


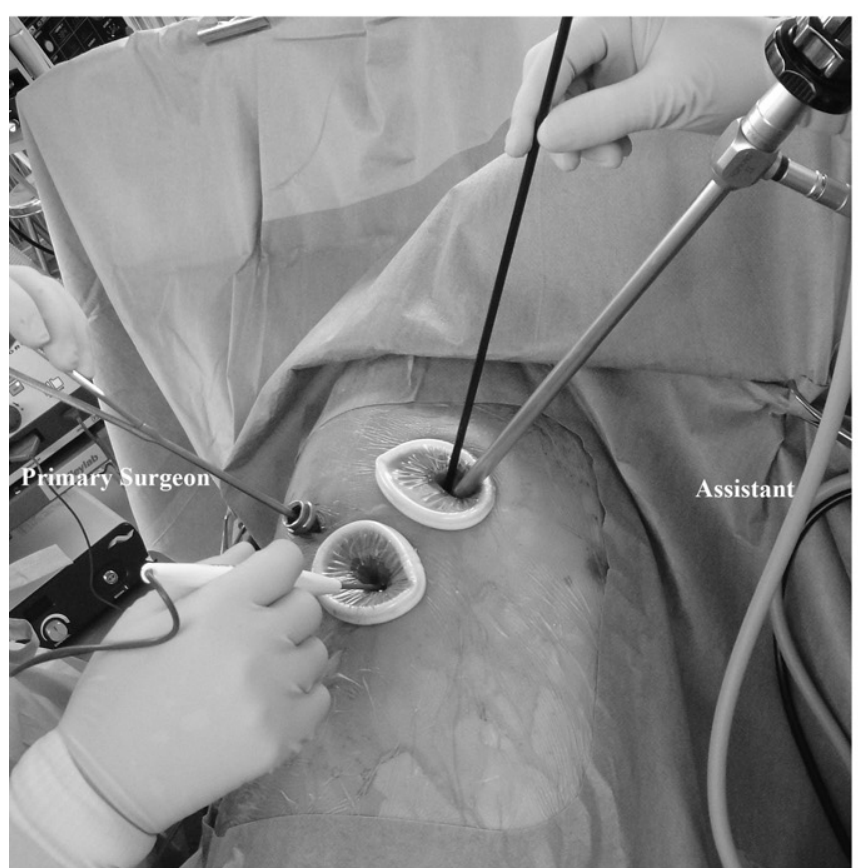

A

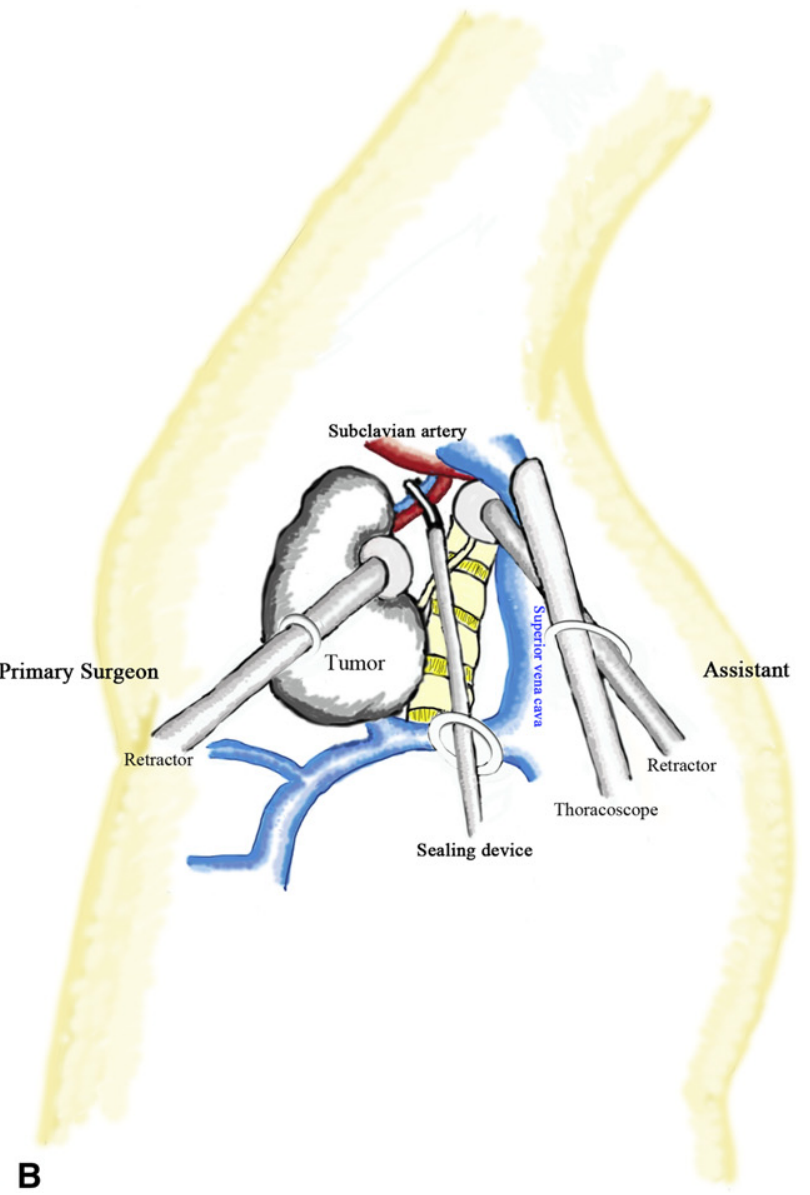

FIGURE 1. Our method of 3-port thoracoscopic surgery. A, Devices are inserted through each port. The primary surgeon stands to the right of the patient and uses 2 proximal access ports, while the assistant inserts 2 instruments through 1 access port. B, Schema shows the feeding vessels being cut by the ultrasonic scalpel in case 1 . The instruments used by the primary surgeon were not interrupted by the thoracoscope or instruments used by the assistant.

dissected from the nerves using ultrasonic scalpels. The operation was completed without any complications. The inferior part of right thyroidal lobe was exposed after the tumor was removed. The operation time was 134 minutes with $3 \mathrm{~mL}$ of intraoperative blood loss. The patient was discharged on postoperative day 2. Pathologic examination confirmed a diagnosis of follicular goiter. A CT scan performed 3 months after the operation showed no recurrence of the tumor and a clip adjacent to thyroid (Figure 2, B).
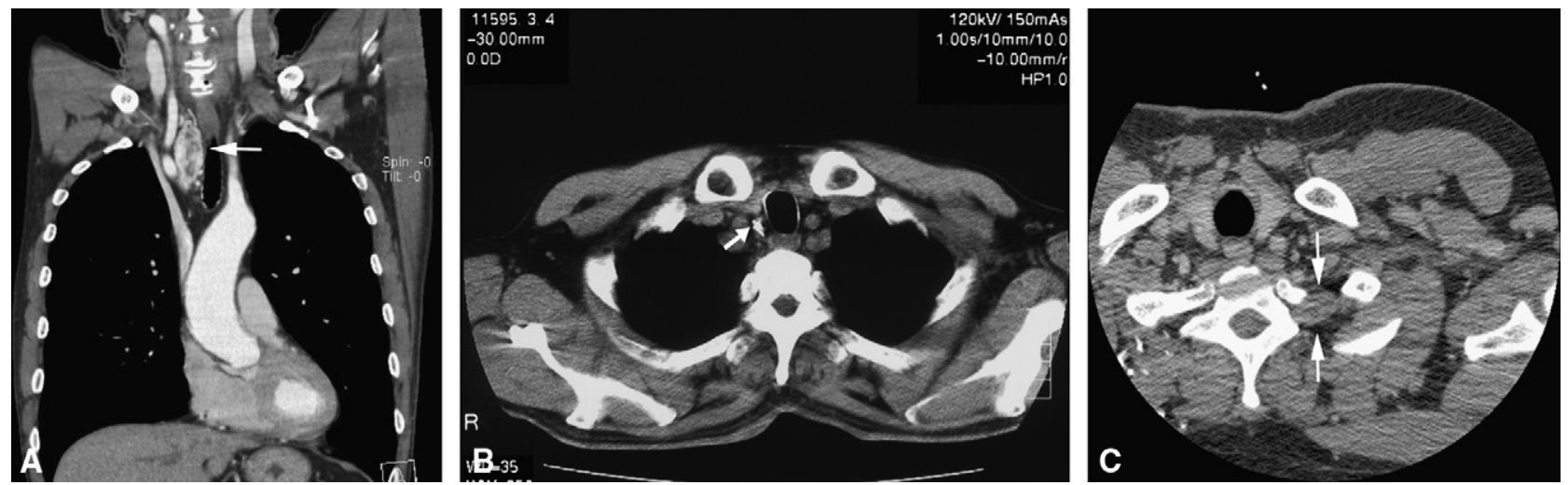

FIGURE 2. Case 1. A, CT scan showed a tumor in the thoracic inlet, extending to the cervical area (white arrow). B, CT performed 3 months after the operation shows a clip adjacent to the thyroid (white arrow). Case 2. C, CT shows a posterior mediastinal tumor extending to the thoracic inlet (white arrow). 


\section{Patient 2}

A 48-year-old man was referred to Yamaguchi University hospital for surgical treatment of a superior mediastinal tumor. A chest CT scan showed that the tumor was located mainly adjacent to the apical thoracic vertebra, but partially above the thoracic inlet (Figure 2, C). Magnetic resonance imaging showed low intensity on the T1-weighted sequence and high intensity on the T2-weighted sequence, which mimicked schwannoma. We attempted to perform totally thoracoscopic resection of the tumor. Careful manipulation was necessary for dissecting the tumor from sympathetic nerve and brachial plexus in the thoracic inlet. We dissected the tumor from the nerves carefully using scissors and an ultrasonic scalpel. The operation was completed totally thoracoscopically with no complications. The operation time was $122 \mathrm{~min}-$ utes, and intraoperative blood loss was $150 \mathrm{~mL}$. The patient was discharged on postoperative day 3. Pathologic examination revealed that the tumor was a schwannoma of the brachial plexus. A CT scan performed 5 months after the operation showed no evidence of recurrence of the tumor.

\section{DISCUSSION}

Resection of an extended superior mediastinal tumor is fraught with technical difficulties because of the anatomic complexity of the superior mediastinum and the thoracic inlet, where there is a large concentration of vital vessels and nerves. Moreover, it is difficult to identify the anatomic detail of the thoracic inlet, even via conventional thoracotomy because it is the farthest and narrowest area to reach; thus, the vagal nerve, recurrent nerve, and sympathetic nerve can be injured during tumor dissection. To avoid these risks, we used a high-definition video system (IMAGE HD, Storz, Germany) with a 30-degree angled scope. This system enabled us to visualize magnified and high-resolution images of individual structures around the thoracic inlet, from the best angles. We also used an ultrasonic scalpel to dissect the tumor without injuring the vital nerves or vessels, because it causes less heat damage than electrocautery to the adjacent structures or organs, ${ }^{3}$ and the narrow blade is useful for dissecting and cutting the structures safely in such a narrow space during thoracoscopic surgery. Apart from these instrumental uses, our strategy is useful for positioning the access ports. Because the thoracoscope and assistant instruments are inserted from the opposite side of the primary surgeon, they rarely interrupt the devices used by the primary surgeon (Figure 1, B).

\section{CONCLUSIONS}

These advantages allow the surgeon to carry out comfortable and less stressful manipulation than that required for conventional thoracotomy for extended superior mediastinal tumors.

\section{References}

1. Shigemura N, Akashi A, Nakagiri T, Matsuda H. VATS with a supraclavicular window for huge substernal goiter: an alternative technique for preventing recurrent laryngeal nerve injury. Thorac Cardiovasc Surg. 2005; 53:231-3.

2. Isowa N, Kikuchi R, Kunimoto Y, Ito K, Tokuyasu H, Fukuda K, et al. Successful resection of posterior mediastinal thyroid cancer by partial sternotomy combined with video-assisted thoracoscopy. Ann Thorac Cardiovasc Surg. 2007;13: 47-9.

3. Takahashi S, Fukuda I, Kuga T, Tanaka M. Exposure of the coronary artery using ultrasonic scalpel. J Thorac Cardiovasc Surg. 2003;125:1533-4.

\title{
A novel technique for identification of the lung intersegmental plane using dye injection into the segmental pulmonary artery
}

\author{
Seiichiro Sugimoto, MD, Takahiro Oto, MD, Kentaroh Miyoshi, MD, and Shinichiro Miyoshi, MD, \\ Okayama, Japan
}

\footnotetext{
From the Department of Cancer and Thoracic Surgery, Okayama University Graduate School of Medicine, Dentistry and Pharmaceutical Sciences, Okayama, Japan. Disclosures: Authors have nothing to disclose with regard to commercial support. Received for publication June 17, 2010; revisions received Aug 1, 2010; accepted for publication Sept 10, 2010; available ahead of print Oct 29, 2010.

Address for reprints: Seiichiro Sugimoto, MD, Department of Cancer and Thoracic Surgery, Okayama University Graduate School of Medicine, Dentistry and Pharmaceutical Sciences, 2-5-1 Shikata Cho, Okayama 700-8558, Japan (E-mail: sugimo-s@cc.okayama-u.ac.jp).

J Thorac Cardiovasc Surg 2011;141:1325-7

$0022-5223 / \$ 36.00$

Copyright (c) 2011 by The American Association for Thoracic Surgery doi:10.1016/j.jtcvs.2010.09.029
}

The diagnostic frequency of small-sized lung cancers has increased with the development and evolution of computed tomography. For small-sized lung cancers, lung segmentectomy is not inferior to lobectomy in selected patients. ${ }^{1,2}$ During lung segmentectomy, the intersegmental line, the 2-dimensional segmental border on the lung surface, is usually identified by inflation/deflation of the target lung segment. ${ }^{3}$ Because regions adjacent to the target lung segment can become inflated through collateral ventilation, it is difficult to identify the intersegmental line in 